\title{
Eslicarbazepine Acetate (BIA 2-093)
}

\author{
Luis Almeida and Patrício Soares-da-Silva \\ Department of Research and Development, BIAL (Portela \& $C^{a}$, SA), S. Mamede do Coronado, Portugal
}

Summary: Eslicarbazepine acetate (ESL) $[(S)-(-)-10$-acetoxy-10,11-dihydro-5H-dibenz[ $b, f]$ azepine-5-carboxamide], formerly known as BIA 2-093, is a novel central nervous system (CNS)-active compound with anticonvulsant activity. It behaves as a voltage-gated sodium channel (VGSC) blocker and is currently under clinical development for the treatment of epilepsy and bipolar disorder. ESL shares with carbamazepine and oxcarbazepine the dibenzazepine nucleus bearing the 5-carboxamide substitute, but is structurally different at the 10,11-position. This molecular variation results in differences in metabolism, preventing the formation of toxic epoxide metabolites such as carbamazepine-10,11 epoxide. In pharmacokinetic studies in humans, ESL was rapidly and extensively metabolized to eslicarbazepine (S-licarbazepine), which is responsible for pharmacological activity. ESL has been tested in patients with refractory partial-onset seizures and was found to be efficacious and well tolerated. Monotherapy studies in adult epileptic patients and add-on studies in epileptic children are in the planning process. The efficacy and safety data appear to be very promising considering the refractory nature of the epileptic population enrolled in studies to date. Results of ongoing phase III studies in adult epileptic patients are expected to be available in 2007 and are required to define the position of ESL in the therapy of patients with epilepsy. Key Words: Eslicarbazepine acetate, BIA 2-093, antiepileptic drugs, drug therapy, epilepsy.

\section{INTRODUCTION}

Eslicarbazepine acetate (ESL) [(S)-(-)-10-acetoxy10,11-dihydro-5H-dibenz[ $b, f]$ azepine-5-carboxamide], formerly known as BIA 2-093, is a novel CNS-active compound currently completing phase III clinical trials as adjunctive therapy in partial epilepsy refractory to standard antiepileptic drugs (AEDs) and undergoing phase II clinical trials as monotherapy in partial epilepsy and in bipolar disorder.

ESL mechanism of action is to block the voltage-gated sodium channel (VGSC). ${ }^{1,2}$ ESL shares with carbamazepine (CBZ) and oxcarbazepine (OXC) the basic chemical structure of a dibenzazepine nucleus with the 5-carboxamide substituent, but is structurally different at the 10,11-position. ${ }^{1,2}$ (FIG. 1). This molecular variation results in differences in ESL metabolism: ESL is reduced by liver esterases to the major active metabolite eslicarbazepine (also known as S-licarbazepine or S-MHD), and, unlike CBZ, is not metabolized to CBZ-10,11-epoxide and is therefore not susceptible to enzyme induction or autoinduction. ${ }^{3}$ ESL also originates R-licarbaz-

Address correspondence and reprint requests to: Patrício Soares-daSilva, BIAL, Department of Research and Development, À Avenida da Siderurgia Nacional, 4745-457 S. Mamede do Coronado, Portugal. E-mail: psoares.silva@bial.com epine and $\mathrm{OXC}$ as minor metabolites by noncytochrome P450 mediated metabolism. ${ }^{3}$ In humans, renal excretion appears to be the major route of elimination of the ESL metabolites.,

The method used for drug assays consists of solid phase extraction followed by high performance liquid chromatography with mass spectrometric detection (LCMS) and has been reported elsewhere. ${ }^{6}$

\section{PHARMACOLOGY}

The precise antiseizure mechanism of action of ESL is unknown. However, in vitro electrophysiological studies indicate that both ESL and its metabolites competitively interact with site 2 of the inactivated state of VGSC. ESL stabilizes the inactive form of the sodium channel, preventing its return to the active state, and sustains repetitive neuronal firing. These actions are thought to be important in the prevention of seizure genesis and spread throughout the intact brain. ESL has a much higher affinity for the inactivated state of the channel compared with the resting state. The affinity of ESL for resting channels is about threefold lower than that of CBZ. This profile suggests that ESL has an enhanced inhibitory selectivity for rapidly firing neurons over those displaying normal activity. ${ }^{7}$ ESL does not appear to interact 


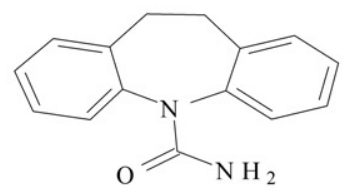

Carbamazepine

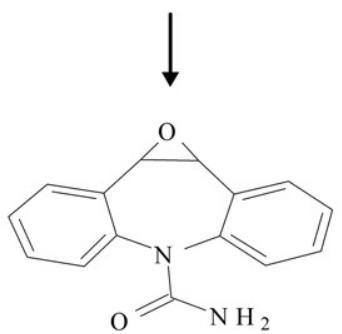

Carbamazepine $-10,11$-epoxide

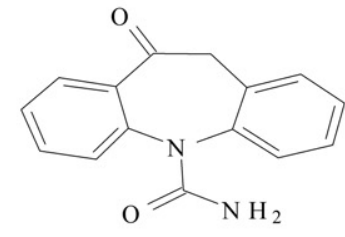

Oxcarbazepine

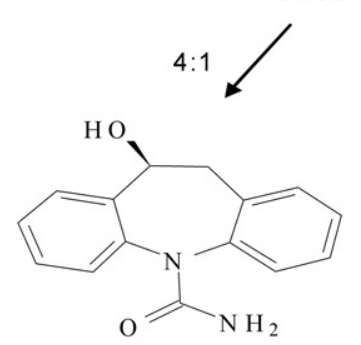

$\mathrm{S}(+)$-licarbazepine

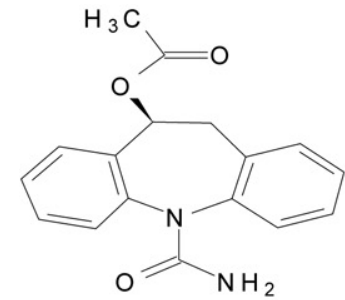

Eslicarbazepine acetate

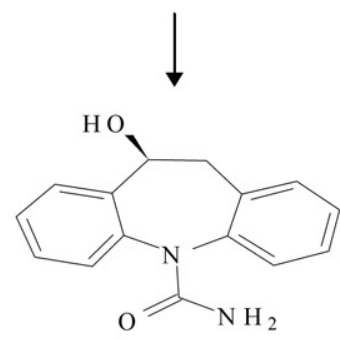

$\mathrm{S}(+)$-licarbazepine

FIG. 1. Chemical structure and main metabolic pathway of carbamazepine, oxcarbazepine, and eslicarbazepine acetate.

with benzodiazepine, GABA, and glutamate receptors. $^{2,8-10}$

In comparison with $\mathrm{CBZ}$ and OXC, ESL was found to cause less neurological impairment to rats after intraperitoneal administration and, consequently, has a higher protective index. ${ }^{1}$ (The protective index is the ratio of the median toxic dose to the median effective dose: PI = $\mathrm{TD}_{50} / \mathrm{ED}_{50}$ ). In a study in cultured hippocampal neurons, ESL was less neurotoxic than CBZ or OXC. ${ }^{11}$

ESL and its metabolites were tested in several models predictive of anticonvulsant efficacy. In the maximal electroshock seizure (MES) test in rat, ESL was found to be equally potent to $\mathrm{CBZ}$ and more potent than $\mathrm{OXC}$ at 2 and $4 \mathrm{~h}$ after administration by gastric tube; however, at $8 \mathrm{~h}$ after administration, the three drugs were equipotent. ESL and CBZ were similarly effective in preventing development of kindling seizures in kindled rats (amygdala kindling in rats mimics complex partial seizures in humans). ESL also showed protective effect against seizures induced by several chemoconvulsants in rats or mice: metrazole, bicuculline, picrotoxin, and 4-aminopyridine (4-AP). ${ }^{12}$

Also evaluated were the effects of oral treatment with ESL on a whole-animal model in which partial seizures can be elicited repeatedly on different days without changes in threshold or seizure patterns. In animals treated with threshold doses of picrotoxin (perfused through the hippocampus), the average number of seizures was $2.3 \pm 1.2$, and average seizure duration was $39.5 \pm 8.4 \mathrm{~s}$. Pretreatment with an ESL dose of $30 \mathrm{mg} / \mathrm{kg}$ $2 \mathrm{~h}$ before picrotoxin microperfusion prevented seizures in $75 \%$ of rats. Lower doses ( 3 and $10 \mathrm{mg} / \mathrm{kg}$ ) did not suppress seizures; however, after administration of 10 $\mathrm{mg} / \mathrm{kg}$, significant reductions in seizure duration (24.3 \pm $6.8 \mathrm{~s})$ and seizure number $(1.6 \pm 0.3)$ were observed. No adverse effects of ESL were observed in the behavioral and EEG patterns studied, including sleep-wakefulness cycle, at the doses studied. ${ }^{13}$

Intrahippocampal microperfusion of latrunculin A (4 $\mu \mathrm{mol} / \mathrm{L}$ ) induces long-term changes in neuronal excitability, leading to the onset of sporadic spontaneous seizures. At oral doses of 10 and $30 \mathrm{mg} / \mathrm{kg}$, ESL resulted in marked attenuation of seizures induced by latrunculin A microperfusion in the rat hippocampus. ${ }^{14}$ The molecular mechanisms behind latrunculin A seizures are still unknown, but the increase in extracellular glutamate concentrations observed during latrunculin A microperfusion are completely reversed by ESL. ${ }^{14}$ This fits well with the observation that ESL is a potent blocker of the 4-aminopyridine- or veratridine-evoked release of glutamate. $^{9}$

\section{Pharmacokinetics and metabolic profile}

When a chiral method is used, the assay can distinguish between eslicarbazepine (S-licarbazepine or $\mathrm{S}-\mathrm{MHD}$ ) and its R-enantiomer (R-licarbazepine or RMHD). Pharmacokinetic studies in humans using such an assay have shown that ESL is rapidly and extensively metabolized to the active metabolite eslicarbazepine (FIG. 1). ${ }^{15-17}$ This metabolite is responsible for approximately $95 \%$ of total systemic drug exposure after oral administration of ESL; R-licarbazepine and OXC are minor metabolites. ${ }^{15-17}$ Plasma levels of parent drug (ESL) usually remained below the limit of quantification.

In some early clinical pharmacokinetic studies, a nonchiral drug assay was used. This method does not allow the separation of S-licarbazepine and R-licarbazepine, and the enantiomeric mixture has been reported as BIA 2-005. In single-dose and multiple-dose studies with ESL in healthy subjects administered with single oral doses 

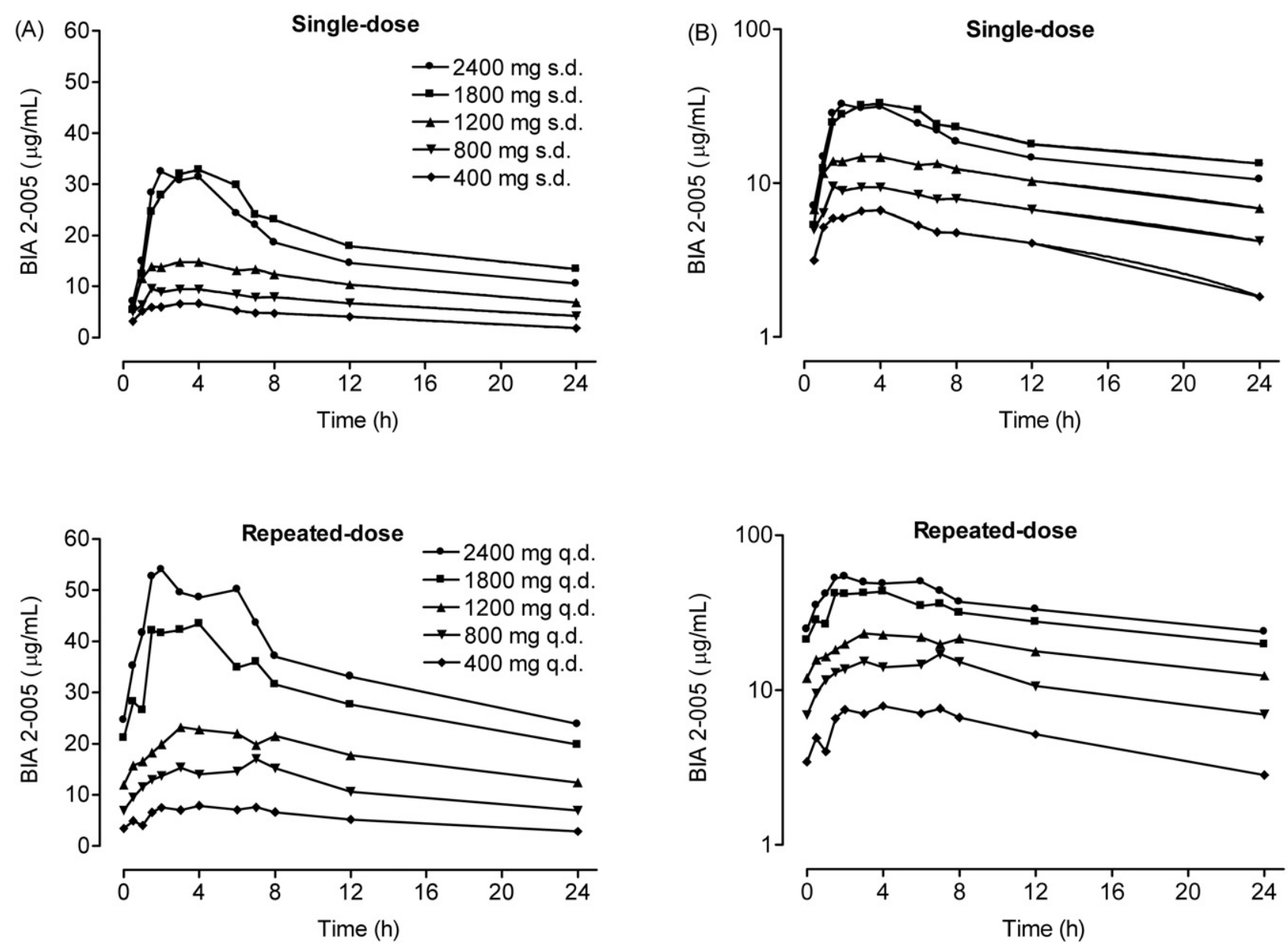

FIG. 2. Mean 24-h plasma concentration-time profiles of the active moiety BIA 2-005 after oral administration of single dose (s.d.) and last dose of an 8-day once-daily (q.d.) regimen of eslicarbazepine acetate to healthy male subjects ( $n=6$ per dose group). (A) Linear scale. (B) Semilog scale.

ranging from $20 \mathrm{mg}$ to $2400 \mathrm{mg}^{4,18}$ and with multipledose studies ranging from $200 \mathrm{mg} 2 \times$ daily (b.i.d.) to $2400 \mathrm{mg} 1 \times$ daily (q.d.), ${ }^{5,18}$ ESL was extensively metabolized to BIA 2-005.

The plasma concentration versus time profiles of BIA
2-005 after single and repeated (8-day treatment) doses are shown in Figure 2. The main pharmacokinetic parameters after a single dose and after the last dose of an 8 -day treatment are given in Tables 1 and 2. Both the rate and the extent of systemic exposure to BIA 2-005 in-

TABLE 1. Main Pharmacokinetic Parameters of BIA 2-005 After Single-Dose Administration of Eslicarbazepine Acetate to Healthy Male Subjects

\begin{tabular}{|c|c|c|c|c|}
\hline Dose $^{*}$ & Mean $C_{\max }, \mu \mathrm{g} / \mathrm{mL}(\% \mathrm{CV})$ & Median $t_{\max }, \mathrm{h}$ (range) & $\begin{array}{c}\text { Mean } \mathrm{AUC}_{0-24 \mathrm{~h}}, \mu \mathrm{g} \cdot \mathrm{h} / \mathrm{mL} \\
(\% \mathrm{CV})\end{array}$ & $\underset{(\% \mathrm{CV})}{\text { Mean apparent }} t_{1 / 2}, \mathrm{~h}$ \\
\hline $20 \mathrm{mg}$ & $0.3(18.7)$ & $0.8(0.5-0.8)$ & $2.4(16.2)$ & $9.1(15.9)$ \\
\hline $50 \mathrm{mg}$ & $0.9(24.7)$ & $0.8(0.5-2)$ & 6.7 (12.7) & $8.1(9.1)$ \\
\hline $100 \mathrm{mg}$ & $1.5(13.8)$ & $1.5(0.5-2)$ & $16.4(11.7)$ & $9.3(8.7)$ \\
\hline $200 \mathrm{mg}$ & $2.9(16.2)$ & $1.5(0.8-2.5)$ & $30.5(23.7)$ & 8.4 (18.8) \\
\hline $400 \mathrm{mg}$ & $5.2(11.6)$ & $4(4-5)$ & $81.5(10.8)$ & $11.7(18.6)$ \\
\hline $600 \mathrm{mg}$ & $8.5(20.0)$ & $4(0.5-5)$ & $119.7(17.4)$ & $12.3(14.8)$ \\
\hline $900 \mathrm{mg}$ & $15.0(18.2)$ & $2.3(0.8-4)$ & $210.3(10.6)$ & $16.3(31.9)$ \\
\hline $1200 \mathrm{mg}$ & $18.6(16.3)$ & $4(2-6)$ & $285.7(16.7)$ & $16.5(6.8)$ \\
\hline $1800 \mathrm{mg}$ & $34.6(16.3)$ & $3.5(3-6)$ & $507.6(17.0)$ & $11.8(11.7)$ \\
\hline $2400 \mathrm{mg}$ & $35.9(42.6)$ & $3(1.5-6)$ & $445.6(26.1)$ & $11.1(21.1)$ \\
\hline
\end{tabular}

$\mathrm{AUC}=$ area under the plasma concentration-time curve over the dosing interval specified in the subscript, $C_{\max }=$ maximum plasma concentrations, $\mathrm{CV}=$ coefficient of variation, $t_{1 / 2}=$ elimination half-life. $* n=6$ per dose group. 
TABLE 2. Main Pharmacokinetic Parameters of BIA 2-005 After Last Dose of an 8-Day Treatment with Eslicarbazepine Acetate in Healthy Male Subjects

\begin{tabular}{lcccc}
\hline \multicolumn{1}{c}{ Dose* } & $\begin{array}{c}\text { Mean } C_{\max }, \\
\mu \mathrm{g} / \mathrm{mL}(\% \mathrm{CV})\end{array}$ & $\begin{array}{c}\text { Median } t_{\max }, \\
\mathrm{h}(\text { range) }\end{array}$ & $\begin{array}{c}\text { Mean } \mathrm{AUC}_{0-24 \mathrm{~h}}, \\
\mu \mathrm{g} \cdot \mathrm{h} / \mathrm{mL}(\% \mathrm{CV})\end{array}$ & $\begin{array}{c}\text { Mean apparent } t_{1 / 2}, \\
\mathrm{~h}(\% \mathrm{CV})\end{array}$ \\
\hline $200 \mathrm{mg}$ b.i.d. & $6.7(23.6)$ & $2.75(1-4)$ & $3.15(12.7)$ & $9.40(16.7)$ \\
$400 \mathrm{mg}$ q.d. & $8.8(16.0)$ & $3(0.5-7)$ & $126.3(11.7)$ & $9.50(18.8)$ \\
$800 \mathrm{mg}$ q.d. & $18.7(14.0)$ & $3.5(1-7)$ & $268.4(10.3)$ & $12.3(22.9)$ \\
$1200 \mathrm{mg}$ q.d. & $25.5(10.8)$ & $3(0.5-6)$ & $423.0(10.9)$ & $13.1(20.1)$ \\
$1800 \mathrm{mg}$ q.d. & $47.7(23.3)$ & $2(0.5-4)$ & $740.3(19.6)$ & $11.3(28.8)$ \\
$2400 \mathrm{mg}$ q.d. & $56.5(20.0)$ & $2(1.5-8)$ & $905.9(12.8)$ & $10.4(24.1)$ \\
\hline
\end{tabular}

AUC $=$ area under the plasma concentration-time curve over the dosing interval specified in the subscript, $C_{\max }=$ maximum plasma concentrations, $\mathrm{CV}=$ coefficient of variation, $t_{\max }=t_{1 / 2}=$ elimination half-life. $* n=6$ per dose group.

creased in an approximately dose-proportional manner after single and repeated administration. BIA 2-005 accumulated in plasma after repeated administration. The mean observed accumulation $\left(R_{\mathrm{o}}\right)$ was 3.0 after $200 \mathrm{mg}$ b.i.d., and 1.4, 1.7, 1.7, 1.5, and 2.1 after 400, 800, 1200, 1800, and $2400 \mathrm{mg}$ q.d, respectively. Steady-state plasma BIA 2-005 concentrations were attained at 4-5 days of q.d. dosing, consistent with an effective elimination half-life $\left(t_{1 / 2}\right)$ on the order of 20-24 h. Renal clearance of BIA 2-005 from plasma was approximately 20-30 mL/min. Renal clearance of BIA 2-005 appeared to be constant over the dose range studied. The total amount of BIA 2-005 recovered in urine was approximately $20 \%$ within $12 \mathrm{~h}$ after the dose and $40 \%$ within 24 hours after. ${ }^{5}$ The dose-proportionality for BIA 2-005 (after single and repeated doses) is in accordance with the concept of linearity regarding its pharmacokinetic behavior (rate and extent of systemic exposure).

The effect of systemic exposure to eslicarbazepine after administration of q.d. and b.i.d. regimens of ESL was investigated in an unpublished study by our group in 11 healthy subjects. That study consisted of 8-day treatment periods separated by a washout period of $10-15$ days. In each treatment period, the volunteers received either an oral dose of ESL $900 \mathrm{mg}$ q.d. or ESL $450 \mathrm{mg}$ b.i.d.. ESL was extensively and rapidly metabolized to eslicarbazepine (S-licarbazepine), the main active metabolite. After the last dose of the ESL $900 \mathrm{mg}$ q.d. regimen, eslicarbazepine maximum plasma concentration $\left(C_{\max }\right)$ was $22.2 \pm 7.3 \mu \mathrm{g} / \mathrm{mL}$ (mean $\pm \mathrm{SD}$ ), and the area under the plasma concentration-time curve over the dosing interval $\left(\mathrm{AUC}_{\tau}\right)$ was $294.0 \pm 58.4 \mu \mathrm{g} \cdot \mathrm{h} / \mathrm{mL}$; after the last dose of the ESL $450 \mathrm{mg}$ b.i.d. regimen, the corresponding values were $C_{\max }=16.7 \pm 4.0 \mu \mathrm{g} / \mathrm{mL}$ and $\mathrm{AUC}_{\tau}=$ $142.1 \pm 25.9 \mu \mathrm{g} \cdot \mathrm{h} / \mathrm{mL}$. The rate of systemic exposure to eslicarbazepine, as assessed by $C_{\max }$, was $33 \%$ higher after ESL $900 \mathrm{mg}$ q.d. in comparison with ESL $450 \mathrm{mg}$ b.i.d. The extent of systemic exposure to eslicarbazepine during a 24-h interval, as assessed by comparing the $\mathrm{AUC}_{\tau}$ of the ESL $900 \mathrm{mg}$ q.d. regimen $\left(\mathrm{AUC}_{0-24 \mathrm{~h}}\right)$ with twice the $\mathrm{AUC}_{\tau}$ of the $\mathrm{ESL} 450 \mathrm{mg}$ b.i.d. regimen $(2 \times$
$\mathrm{AUC}_{0-12 \mathrm{~h}}$ ), was 3\% higher after ESL $900 \mathrm{mg}$ q.d. than after ESL $450 \mathrm{mg}$ b.i.d.

A study showed that oral suspension $50 \mathrm{mg} / \mathrm{mL}$ and tablets strengths $200 \mathrm{mg}$ and $800 \mathrm{mg}$ are bioequivalent. ${ }^{6}$

The effect of age and gender on the pharmacokinetics of ESL was investigated in a study in 12 young (18-40 years) and 12 elderly (65 years or more) healthy subjects. ${ }^{15,17}$ In each age group, six subjects were female and six were male. That study consisted of a single-dose period (600 mg; phase A) and a multiple-dose period (600 mg q.d. for 8 days; phase B), separated by 4 days. Plasma concentration-time profiles of eslicarbazepine after phase A single dose and phase B last dose are presented in Figure 3. After a 600-mg single dose, mean $C_{\max }$ and AUC from 0 to infinity $\left(\mathrm{AUC}_{0-\infty}\right)$ were, respectively, $9.9 \mu \mathrm{g} / \mathrm{mL}$ and $180.9 \mu \mathrm{g} \cdot \mathrm{h} / \mathrm{mL}$ in young subjects and $9.5 \mu \mathrm{g} / \mathrm{mL}$ and $196.0 \mu \mathrm{g} \cdot \mathrm{h} / \mathrm{mL}$ in elderly subjects, and a corresponding $9.3 \mu \mathrm{g} / \mathrm{mL}$ and 171.9 $\mu \mathrm{g} \cdot \mathrm{h} / \mathrm{mL}$ in men and $10.1 \mu \mathrm{g} / \mathrm{mL}$ and $205.0 \mu \mathrm{g} \cdot \mathrm{h} / \mathrm{mL}$ in women. After multiple dosing, steady-state plasma concentrations were attained at 4-5 days of administration in both age and sex groups, consistent with an effective half-life in the order of 17-18 h.

After the last dose of phase $\mathrm{B}$, mean $C_{\max }$ and $\mathrm{AUC}_{0-\infty}$ of eslicarbazepine were, respectively, $17.3 \mu \mathrm{g} / \mathrm{mL}$ and $296.7 \mu \mathrm{g} \cdot \mathrm{h} / \mathrm{mL}$ in young subjects and $15.1 \mu \mathrm{g} / \mathrm{mL}$ and $294.3 \mu \mathrm{g} \cdot \mathrm{h} / \mathrm{mL}$ in elderly subjects, and a corresponding $15.5 \mu \mathrm{g} / \mathrm{mL}$ and $295.8 \mu \mathrm{g} \cdot \mathrm{h} / \mathrm{mL}$ in men and 16.8 $\mu \mathrm{g} / \mathrm{mL}$ and $295.2 \mu \mathrm{g} \cdot \mathrm{h} / \mathrm{mL}$ in women. After the single dose (phase A), the eslicarbazepine $C_{\max }, \mathrm{AUC}_{0-24}$, and $\mathrm{AUC}_{0-\infty}$ elderly/young geometric mean ratio (GMR) with $95 \%$ confidence interval $(95 \% \mathrm{CI})$ was, respectively, $0.95(0.81,1.14), 1.02(0.86,1.24)$, and 1.06 $(0.88,1.32)$; the corresponding female/male GMR $(95 \%$ CI) was $1.09(0.87,1.43), 1.16(0.95,1.48)$, and 1.17 $(0.90,1.63)$. After the last dose of phase $\mathrm{B}$, the eslicarbazepine $C_{\max }, \mathrm{AUC}_{0-24}$, and $\mathrm{AUC}_{0-\infty}$ elderly/young GMR (95\% CI) was, respectively, $0.88(0.77,1.03), 0.98$ $(0.90,1.09)$, and $1.01(0.89,1.18)$; the corresponding female/male GMR $(95 \% \mathrm{CI})$ was $1.10(0.98,1.27), 1.04$ $(0.88,1.28)$, and $1.01(0.83,1.30)$. It was concluded that 
(A)

Post single-dose of Phase A

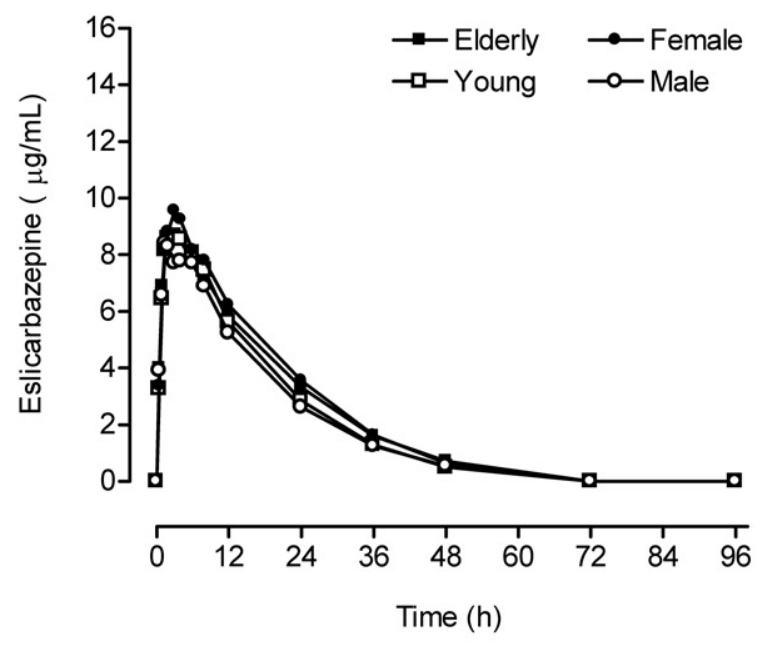

Post last dose of Phase B

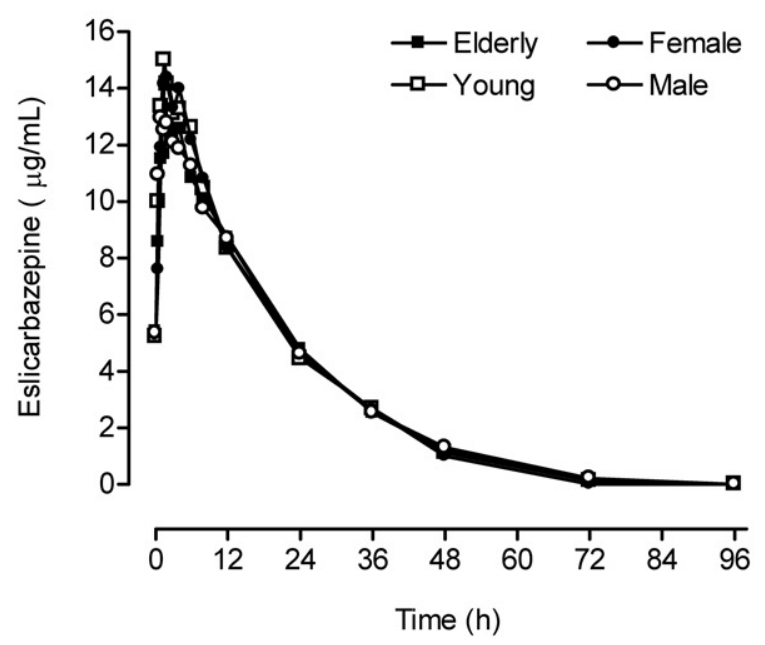

(B)

Post single-dose of Phase A

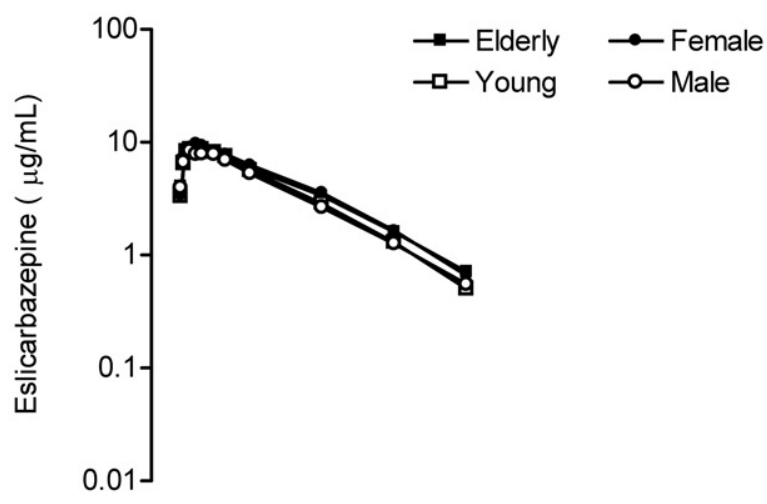

Post last dose of Phase B

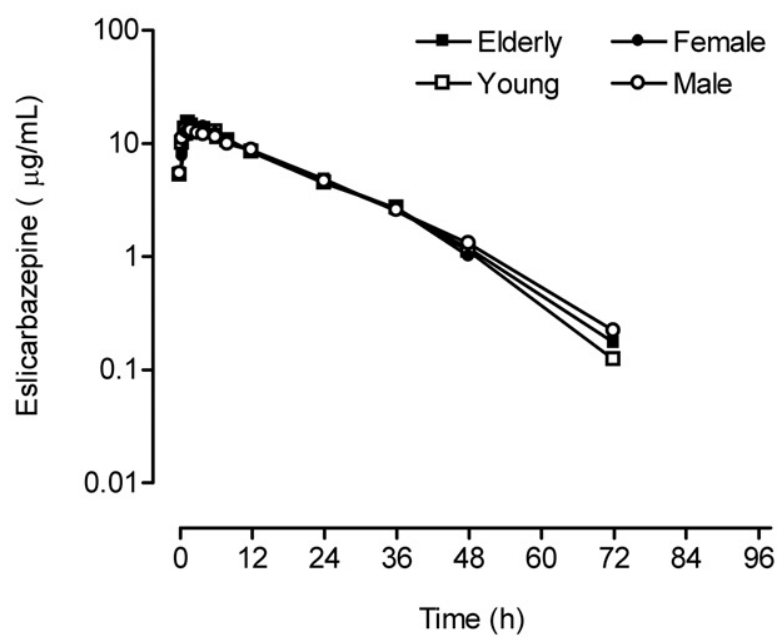

FIG. 3. Mean plasma concentration-time profile of the active moiety eslicarbazepine after a 600 -mg single dose (phase A) and after the last dose of an 8-day once-daily 600-mg dose regimen (phase B) of eslicarbazepine acetate to young and elderly healthy subjects ( $n$ $=12$ per age group; $n=12$ per gender group). (A) Linear scale. (B) Semilog scale.

no dosage adjustment on the basis of age or gender is necessary. 15,17

\section{Drug interactions}

In vitro studies with human plasma showed that the binding of eslicarbazepine to plasma proteins is relatively low (30\%) and is not affected by the presence of warfarin, diazepam, digoxin, phenytoin, and tolbutamide, and that the binding of warfarin, diazepam, digoxin, phenytoin, and tolbutamide is not affected by the presence of eslicarbazepine (unpublished observations).

The interaction of eslicarbazepine with other drugs and enzymes has been also evaluated in vitro. Eslicarbazepine appeared to have no relevant effect on the activity of the cytochrome P450 (CYP) isoforms CYP1A2, CYP2A6, CYP2B6, CYP2C9, CYP2C19, CYP2D6, CYP2E1, CYP3A4, and CYP4A9/11; the uridine diphosphate-5' -glucuronosyltransferases (UGT) UGT1A1 and UGT1A6; and the epoxide hydrolase (EH) of human hepatic microsomes. The most significant effect was a moderate inhibition (38\% reduction) of CYP2C9-mediated tolbutamide 4-hydroxylation and a mild activation (39\% increase) of UGT1A1-mediated ethinylestradiol glucuronidation (unpublished observations). Another study investigated the potential for eslicarbazepine to induce hepatic drug metabolizing enzymes using freshly isolated human hepatocytes (unpublished observations). Those in vitro findings suggested that it is unlikely that 
eslicarbazepine will significantly induce the metabolism of drugs coadministered during clinical use that undergo metabolism through CYP1A2, CYP3A4, and phase 2 hepatic enzymes.

The effect of food on the ESL pharmacokinetics was investigated in a randomized, two-way crossover study in 12 healthy subjects. ${ }^{19}$ That study consisted of two consecutive treatment periods separated by a washout of 14 days or more. In each of the study periods, subjects were administered a single dose of ESL $800 \mathrm{mg}$ after either a standard high-fat content meal or $10 \mathrm{~h}$ of fasting. The nonchiral assay was used, and therefore the active moiety was reported as BIA 2-005. The BIA 2-005 $C_{\max }$ was $12.8 \pm 1.8$ (mean $\pm \mathrm{SD}$ ) in fed (or Test) conditions and $11.3 \pm 1.9 \mu \mathrm{g} / \mathrm{mL}$ in fasting (or Reference) conditions; the corresponding $\mathrm{AUC}_{0-\infty}$ was $242.5 \pm 32.1$ (Test) and $243.6 \pm 31.1 \mu \mathrm{g} \cdot \mathrm{h} / \mathrm{mL}$ (Reference). The point estimate and $90 \%$ confidence interval, or PE $(90 \%$ CI), of the Test/Reference $C_{\max }$ GMR was 1.14 (1.04, 1.25); the PE (90\% CI) for the $\mathrm{AUC}_{0-\infty}$ Test/Reference ratio was $1.00(0.95,1.04)$. Bioavailability of ESL administered in fed and fasting conditions was similar, and bioequivalence was accepted for both $\mathrm{AUC}_{0-\infty}$ and $C_{\max }$ because the $90 \%$ CI lies within the usual acceptance range of $0.80,1.25 .^{20}$ No statistically significant differences were found in $t_{\max }$ (i.e., time of occurrence of $C_{\text {max }}$ ).

The effect of ESL on the digoxin pharmacokinetics was investigated in a randomized, double-blind, placebocontrolled, two-way crossover study in 12 healthy subjects. ${ }^{21}$ That study consisted of two 8-day treatment periods separated by a washout of 10 or more days. During each treatment period, subjects received either a daily oral dose of ESL $1200 \mathrm{mg}$ q.d. or a placebo (PLA) concomitantly with a digoxin q.d. dose of $0.5 \mathrm{mg} /$ day on days 1 and 2 , and $0.25 \mathrm{mg}$ /day on days $3-8$. Minimum (predose) serum digoxin concentrations $\left(C_{\min }\right)$ at days 6 , 7 , and 8 were, respectively, 445, 452, and $633 \mathrm{pg} / \mathrm{mL}$ with PLA and 475,522 , and $561 \mathrm{pg} / \mathrm{mL}$ with ESL. The PE $(90 \% \mathrm{CI})$ values of PLA/ESL $C_{\max }$ and $\mathrm{AUC}_{0-24}$ were $0.85(0.68,1.07)$ and $0.96(0.90,1.03)$, respectively. The observed $15 \%$ decrease in $C_{\max }$ of digoxin when digoxin was administered concomitantly with ESL is not expected to affect digoxin efficacy, because the extent of exposure (as assessed by $\mathrm{AUC}_{0-24}$ ) was similar. It was concluded that, at the dose of $1200 \mathrm{mg}$ q.d., ESL had no relevant effect on the steady-state extent of systemic exposure to digoxin.

The effect of ESL on the warfarin pharmacokinetics and pharmacodynamics was investigated in a multipledose, open-label, single-period study consisting of three consecutive phases:

Phase A: run-in warfarin dose-finding phase, aimed at identifying the warfarin daily dose that stabilizes the INR between 1.3 and 1.8 (minimum of 16 days and up to 21 days).

Phase B: warfarin pharmacokinetics and international normalized ratio (INR) profiling before, during, and after a 7-day multiple-dose treatment with ESL in which subjects received ESL $1200 \mathrm{mg}$ q.d. concomitantly with their individualized dose of warfarin defined in the run-in phase $\mathrm{A}$, the aim of which was to assess whether ESL affects INR and levels of warfarin when added to concomitant warfarin therapy.

Phase C: a 7-day period in which subjects received warfarin alone at their individualized doses, aimed at assessing whether ESL affected INR and levels of warfarin when it was removed from concomitant warfarin therapy.

The $C_{\max }$ of $\mathrm{S}$-warfarin was reached at a time $t_{\max }$ between 1.0 and $4.0 \mathrm{~h}$ after the dose (median of $1.0 \mathrm{~h}$ ). Thereafter, plasma S-warfarin concentrations declined in a multiphasic manner with a mean apparent terminal $t_{1 / 2}$ of $27.0 \mathrm{~h}$ (day 1 of phase B) and $24.5 \mathrm{~h}$ (day 8 of phase B). Systemic exposure $\left(C_{\max }\right.$ and $\left.\mathrm{AUC}_{0-24}\right)$ to S-warfarin decreased after administration of ESL concomitantly with warfarin. The PE $(90 \% \mathrm{CI})$ of the $\mathrm{AUC}_{0-24}$ ratio of Test (warfarin plus ESL: day 8 of phase B) over Reference (= warfarin alone: day 1 of phase B) was 0.77 $(0.72,0.82)$. For the $C_{\max }$ ratio, PE was 0.81 and $90 \% \mathrm{CI}$ was $0.76,0.86$. No statistical difference was found between $t_{\max }$ values. $C_{\max }$ of R-warfarin was reached $t_{\max }$ between 1.0 and $4.0 \mathrm{~h}$ after the dose (median of $1.0 \mathrm{~h}$ ) at day 1 of phase $B$ and between 1.0 and $8.0 \mathrm{~h}$ after the dose (median of $1.0 \mathrm{~h}$ ) at day 8 . Thereafter, plasma R-warfarin concentrations declined in a multiphasic manner, with a mean $t_{1 / 2}$ of $33.7 \mathrm{~h}$ on day 1 of phase $\mathrm{B}$ and $31.8 \mathrm{~h}$ on day 8. Systemic exposure $\left(C_{\max }\right.$ and $\left.\mathrm{AUC}_{0-24}\right)$ to R-warfarin did not significantly change after administration of ESL concomitantly with warfarin. The PE $(90 \% \mathrm{CI})$ was 0.98 $(0.92,1.04)$ for $\mathrm{AUC}_{0-24}$ and $0.97(0.91,1.02)$ for $C_{\max }$. No statistical difference was found between $t_{\max }$ values for R-warfarin.

During the three last days of phase A (warfarin, administered alone) and of phase B (ESL administered with warfarin), the INR values were $1.45 \pm 0.10$ (mean \pm SD) and $1.51 \pm 0.25$, respectively. The small increase of $4.0 \%$ in the INR value appears to have no clinical or statistical significance $(90 \% \mathrm{CI}:-1.03,9.12)$ and thus we conclude that there was no significant pharmacodynamic interaction between ESL and warfarin. In phase C, after discontinuation of ESL administration, a slight INR decrease was found: $-5.42 \%$ (90\% CI: $-8.85,-1.98$ ).

In an unpublished study by our group, administration of ESL $1200 \mathrm{mg}$ q.d. concomitantly with a hormonal oral contraceptive containing $30 \mu \mathrm{g}$ ethinylestradiol and 150 

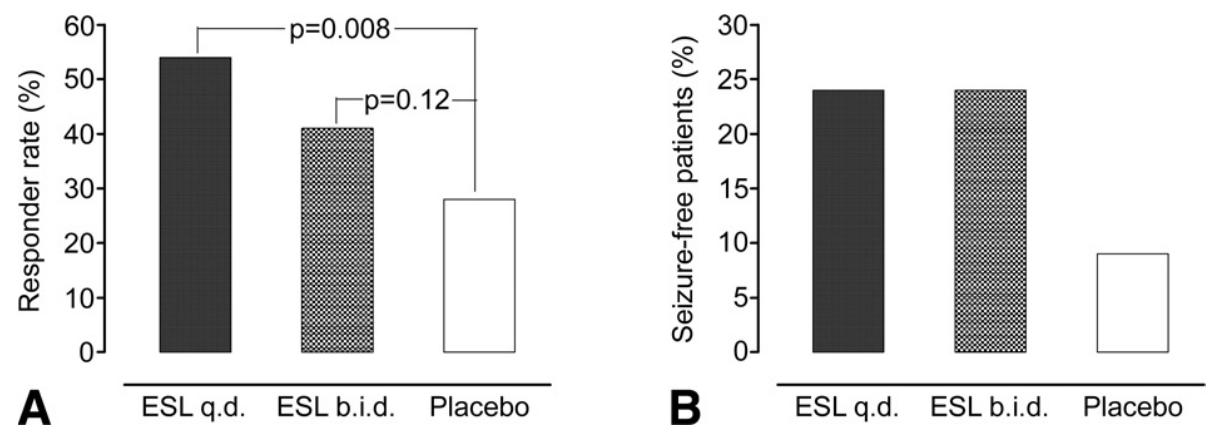

FIG. 4. A, Responder rate (i.e., proportion of patients with at least $50 \%$ decrease in number of seizures) and; B, proportion of seizure-free patients in a phase II study with eslicarbazepine acetate (ESL) in patients with refractory partial-onset epilepsy. q.d. = ESL up to $1200 \mathrm{mg}$ once daily, b.i.d. = ESL up to $600 \mathrm{mg}$ twice daily.

$\mu \mathrm{g}$ levonorgestrel was shown to decrease the plasma concentrations of the two hormonal components when Test (ESL plus oral contraceptive) and Reference (oral contraceptive alone) were compared. In this study, mean levonorgestrel $C_{\max }$ decreased $13 \%$ (Test/Reference PE: 0.87; 90\% CI: $0.79,0.95)$ and $\mathrm{AUC}_{0-24}$ decreased $24 \%$ (PE: 0.76; 90\% CI: 0.68, 0.86) after administration of ESL. In a published study with OXC, the reported decrease in the AUC of levonorgestrel was $47 \% .^{22}$ In the ESL study, mean $C_{\max }$ ethinylestradiol decreased $20 \%$ (PE: 0.80; 90\% CI: 0.79-0.95) and $\mathrm{AUC}_{0-24}$ decreased 32\% (PE: 0.76; 90\% CI: 0.68-0.86) after administration of ESL. With OXC, the reported decrease in the AUC of ethinylestradiol was $47 \% .^{22}$ No statistically significant difference was found between $t_{\max }$ values for levonorgestrel and ethinylestradiol after administration of ESL.

CYP3A4 in the liver is responsible for the 2-hydroxylation of ethinylestradiol, the main route of elimination of the steroid. ${ }^{23}$ The 2-hydroxy metabolite is further transformed by methylation and glucuronidation prior to urinary and fecal excretion. Ethinylestradiol is excreted in the urine and feces as glucuronide and sulfate conjugates, and undergoes enterohepatic circulation. The metabolism of levonorgestrel is more complex than that of ethinylestradiol: it involves reduction of the unsaturated ketone ring $\mathrm{A}$ as well as 2- and 6-hydroxylation, followed by conjugation. ${ }^{24}$ Most of the metabolites that circulate in the blood are sulfates, whereas excretion occurs predominantly in the form of glucuronides.

Because a significant proportion of ethinylestradiol and levonorgestrel metabolism occurs during the first pass in the liver, induction of metabolism provides an explanation not only for the decrease in extent of exposure (as assessed as AUC) but also for the reduced peak plasma concentration (assessed as $C_{\max }$ ) after absorption. ${ }^{24}$ The mostly likely explanation is that the decrease in plasma ethinylestradiol and levonorgestrel was due to induction of the CYP3A4 isoenzymes involved in their oxidation. $^{22}$ Although the decrease in the extent of exposure to levonorgestrel and ethinylestradiol after ESL was approximately half that reported with $\mathrm{OXC},{ }^{22}$ it may be concluded that concomitant administration of ESL and hormonal contraceptives can render these contraceptives less effective.

In a phase II study in patients (unpublished observations), administration of ESL $400 \mathrm{mg}, 800 \mathrm{mg}$, and 1200 mg q.d or divided in two doses (b.i.d.) did not significantly affect the mean trough serum concentrations of valproate. The concomitant administration of ESL q.d. did not affect the lamotrigine concentrations over time, but when ESL was administered b.i.d., a significant decrease in the lamotrigine concentrations was found. Topiramate concentrations were significantly affected by the concomitant administration of ESL (q.d. or b.i.d.), but the decrease was more marked in the b.i.d. dosing. However, these results must be considered merely exploratory, and further and more reliable data will come from the currently ongoing phase III clinical trial program.

\section{Efficacy data}

Results are available for a multicenter, double-blind, randomized, placebo-controlled study in 143 patients with at least four partial-onset seizures per month in spite of treatment with one or two AEDs. ${ }^{25}$ That study consisted of a 12-week treatment period followed by a 1-week tapering off. Patients were randomly assigned to one of three groups: treatment with ESL q.d. $(n=50)$, ESL b.i.d. $(n=46)$, or placebo (PLA, $n=47)$. For the first 4 weeks, daily doses were $400 \mathrm{mg}$; doses were then titrated up to $800 \mathrm{mg}$ and $1200 \mathrm{mg}$ at 4-week intervals. Statistical analysis was performed in the intent-to-treat population (all randomized patients with at least one administration of study medication). Proportion of responders (patients with a $\geq 50 \%$ reduction in seizure frequency) had been defined as the primary endpoint.

The main efficacy results are presented in Figure 4. The percentage of responders at the end of the treatment period versus baseline showed a statistically significant difference between ESL 1200 mg q.d. and PLA groups (54\% versus $28 \% ; P=0.008$ ); the difference between the ESL $600 \mathrm{mg}$ b.i.d. (41\%) and PLA did not reach 
TABLE 3. Summary of Treatment-Emergent Adverse Events in a Phase II Study with Eslicarbazepine Acetate in Epileptic Patients

\begin{tabular}{|c|c|c|c|}
\hline & Eslicarbazepine acetate q.d. & Eslicarbazepine acetate b.i.d. & Placebo \\
\hline Sample size, no. of patients & 50 & 46 & 47 \\
\hline Any AE, no. (\%) & $19(38.0)$ & $19(41.3)$ & $21(44.7)$ \\
\hline Discontinued due to AE, no. (\%) & $3(6.0)$ & $4(8.7)$ & $4(8.5)$ \\
\hline Discontinued due to SFI, no. (\%) & $0(0.0)$ & $2(4.3)$ & $1(2.1)$ \\
\hline \multicolumn{4}{|c|}{$\begin{array}{l}\text { AEs possibly related to treatment and occurring } \\
\text { in }>1 \text { patient, no. }(\%)\end{array}$} \\
\hline Headache & $1(2.0)$ & $4(8.7)$ & $1(2.1)$ \\
\hline Dizziness & $1(2.0)$ & $3(6.5)$ & $0(0.0)$ \\
\hline Nausea & $2(4.0)$ & $3(6.5)$ & $2(4.3)$ \\
\hline Somnolence & $3(6.0)$ & $1(2.2)$ & $0(0.0)$ \\
\hline Vomiting & $0(0.0)$ & $3(6.5)$ & $0(0.0)$ \\
\hline Hair loss & $1(2.0)$ & $2(4.3)$ & $0(0.0)$ \\
\hline Dry mouth & $0(0.0)$ & $2(4.3)$ & $0(0.0)$ \\
\hline Concentration impaired & $0(0.0)$ & $2(4.3)$ & $0(0.0)$ \\
\hline Insomnia & $0(0.0)$ & $0(0.0)$ & $2(4.3)$ \\
\hline CK increase & $1(2.0)$ & $0(0.0)$ & $1(2.1)$ \\
\hline Drowsiness & $1(2.0)$ & $0(0.0)$ & $1(2.1)$ \\
\hline
\end{tabular}

Some subjects reported more than one adverse event.

$\mathrm{AE}=$ adverse event, b.i.d. $=$ twice daily, $\mathrm{CK}$, creatine kinase, q.d. $=$ once daily, $\mathrm{SFI}=$ seizure frequency increase.

statistical significance $(P=0.12)$. During weeks $1-4$ $(400 \mathrm{mg} /$ day $)$, no significant differences in responder rate were found. During weeks $5-8(800 \mathrm{mg} /$ day $)$, the proportion of responders reached $58 \%$ in the ESL q.d., compared with $38 \%$ in the PLA groups $(P=0.04)$; no statistical difference was found between the ESL b.i.d. and PLA groups $(P=0.36)$. A significantly higher proportion of responders in weeks 5-8 was found in the ESL q.d. group, compared with the ESL b.i.d. groups (58.0 versus $32.6 \%$, respectively, $P=0.022$ ). In each of the three 4-week periods, the ESL q.d. group showed a significantly higher reduction in seizure number than the ESL b.i.d. group $(P=0.037,0.018$, and 0.002 , respectively).

Results from three ongoing phase III placebo-controlled studies in refractory adult epileptic patients, which are expected to be available in 2007, will define the position of ESL in the therapy of patients with epilepsy. Other ongoing or planned studies include phase II/III studies in epileptic children, monotherapy studies in adult epileptic patients, and phase II studies in acute mania and recurrence prevention in bipolar disorder.

\section{Exposure, tolerability, and side-effects}

More than 1800 subjects have been enrolled in ESL clinical studies performed to date or currently ongoing, and more than 1200 of these have been exposed to single or repeated doses of ESL. In human pharmacology studies in healthy subjects, the highest dosage used was 2400 mg q.d. for 8 days. ${ }^{18}$ In add-on studies in epileptic patients, ESL has been used in doses up to $1200 \mathrm{mg} /$ day concomitantly with one or two antiepileptic drugs in standard therapeutic doses. ${ }^{25}$ The longest exposure to
ESL as add-on therapy in epileptic patients is approximately 20 months.

In human single-dose and multiple-dose pharmacology studies up to $2400 \mathrm{mg}$ in healthy subjects, ESL was generally well tolerated. ${ }^{4,5,18}$ Adverse events were generally mild in severity. The most commonly reported adverse events were headache, somnolence, dizziness, and paresthesia circumoral, lips, or tongue. In a study in elderly versus young subjects, all healthy, no significant difference in tolerability was found between elderly and young. ${ }^{15}$ No drug-related serious adverse events were reported. No abnormal trends nor abnormal vital signs were found in the safety clinical laboratory tests. There were no clinically significant electrocardiographic abnormalities, nor was there evidence of QT interval prolongation.

In a phase II study in epileptic patients (TABLE 3), the proportion of patients reporting at least one adverse event and the incidence of adverse events was not significantly different between the ESL q.d., ESL b.i.d., and placebo groups. ${ }^{25}$ The most reported adverse events were nausea, headache, dizziness, and somnolence. Most adverse events were mild in intensity and no drug-related serious adverse events occurred. No abnormal trends in the safety clinical laboratory tests or in vital signs were found.

\section{REFERENCES}

1. Benes J, Parada A, Figueiredo AA, et al. Anticonvulsant and sodium channel-blocking properties of novel 10,11-dihydro-5H dibenz $[b, f]$ azepine-5-carboxamide derivatives. J Med Chem 1999; 42:2582-2587.

2. Parada A, Soares-da-Silva P. The novel anticonvulsant BIA 2-093 inhibits transmitter release during opening of voltage-gated sodium 
channels: a comparison with carbamazepine and oxcarbazepine. Neurochem Int 2002;40:435-440.

3. Bialer M. New antiepileptic drugs that are second generation to existing antiepileptic drugs. Expert Opin Investig Drugs 2006;15: 637-647.

4. Almeida L, Soares-da-Silva P. Safety, tolerability and pharmacokinetic profile of BIA 2-093, a novel putative antiepileptic agent, during first administration to humans. Drugs R D 2003;4:269-284.

5. Almeida L, Soares-da-Silva P. Safety, tolerability, and pharmacokinetic profile of BIA 2-093, a novel putative antiepileptic, in a rising multiple-dose study in young healthy humans. J Clin Pharmacol 2004;44:906-918.

6. Fontes-Ribeiro C, Nunes T, Falcão A, et al. Eslicarbazepine acetate (BIA 2-093): relative bioavailability and bioequivalence of 50 $\mathrm{mg} / \mathrm{mL}$ oral suspension and $200 \mathrm{mg}$ and $800 \mathrm{mg}$ tablet formulations. Drugs R D 2005;6:253-260.

7. Bonifacio MJ, Sheridan RD, Parada A, Cunha RA, Patmore L, Soares-da-Silva P. Interaction of the novel anticonvulsant, BIA 2-093, with voltage-gated sodium channels: comparison with carbamazepine. Epilepsia 2001;42:600-608.

8. Ambrosio AF, Silva AP, Araujo I, et al. Neurotoxic/neuroprotective profile of carbamazepine, oxcarbazepine and two new putative antiepileptic drugs, BIA 2-093 and BIA 2-024. Eur J Pharmacol 2000;406:191-201.

9. Ambrosio AF, Silva AP, Malva JO, Soares-da-Silva P, Carvalho AP, Carvalho CM. Inhibition of glutamate release by BIA 2-093 and BIA 2-024, two novel derivatives of carbamazepine, due to blockade of sodium but not calcium channels. Biochem Pharmacol 2001;61:1271-1275.

10. Cunha RA, Coelho JE, Costenla AR, et al. Effects of carbamazepine and novel 10,11-dihydro-5H-dibenz[ $b, f]$ azepine-5-carboxamide derivatives on synaptic transmission in rat hippocampal slices. Pharmacol Toxicol 2002;90:208-213.

11. Araujo IM, Ambrosio AF, Leal EC, et al. Neurotoxicity induced by antiepileptic drugs in cultured hippocampal neurons: a comparative study between carbamazepine, oxcarbazepine, and two new putative antiepileptic drugs, BIA 2-024 and BIA 2-093. Epilepsia 2004;45:1498-1505.

12. Bialer M, Johannessen S, Kupferberg HJ, Levy RH, Perucca E, Tomson T. Progress report on new antiepileptic drugs: a summary of the Seventh EILAT Conference (EILAT VII). Epilepsy Res 2004;61:1-48.

13. Sierra-Paredes G, Oreiro-Garcia MT, Nuñez-Rodriguez A, Vazquez-Lopez A, Sierra-Marcuño G. Effect of novel anticonvul- sant BIA 2-093 on seizures induced by picrotoxin microperfusion in the rat hippocampus. Epilepsia 2004;45(Suppl 3):116.

14. Sierra-Marcuño G, Oreiro-García MT, Vázquez-Illanes MD, Sierra-Paredes G. Effect of novel anticonvulsant BIA 2-093 on latrunculin A-induced seizures and extracellular glutamate concentrations in the rat hippocampus. Epilepsia 2005;46(Suppl 8):302. Abstract.

15. Almeida L, Falcão A, Maia J, Mazur D, Gellert M, Soares-da-Silva P. Single-dose and steady-state pharmacokinetics of eslicarbazepine acetate (BIA 2-093) in healthy elderly and young subjects. J Clin Pharmacol 2005;45:1062-1066.

16. Silveira P, Falcão A, Almeida L, Maia J, Soares-da-Silva P. BIA 2-093 pharmacokinetics in healthy elderly subjects. Epilepsia 2004;45(Suppl 3):157. Abstract.

17. Soares-da-Silva P, Almeida L, Falcão A, Maia J. Effect of age and gender on the pharmacokinetics of eslicarbazepine acetate. Epilepsia 2005;46(Suppl 8):216-217. Abstract.

18. Vaz-da-Silva M, Nunes T, Soares E, et al. Eslicarbazepine acetate pharmacokinetics after single and repeated doses in healthy subjects. Epilepsia 2005;46(Suppl 8):191. Abstract.

19. Maia J, Vaz-da-Silva M, Almeida L, et al. Effect of food on the pharmacokinetic profile of eslicarbazepine acetate (BIA 2-093). Drugs R D 2005;6:201-206.

20. EMEA-CPMP. Note for guidance on the investigation of bioavailability and bioequivalence. Presented at the European Agency for the Evaluation of Medicinal Products-Committee for Proprietary Medicinal Products, CPMP/EWP/QWP/1401/9; Jul 26, 2001. Available at: http://www.emea.eu.int/pdfs/human/ewp/140198en. pdf.

21. Maia J, Almeida L, Vaz-da-Silva M, et al. Effect of eslicarbazepine acetate on the steady-state pharmacokinetics of digoxin in healthy subjects. Epilepsia 2005;46(Suppl 8):178-179. Abstract.

22. Fattore C, Cipolla G, Gatti G, et al. Induction of ethinylestradiol and levonorgestrel metabolism by oxcarbazepine in healthy women. Epilepsia 1999;40:783-787.

23. Back DJ, Orme MLE. Pharmacokinetic drug interactions with oral contraceptives. Clin Pharmacokinet 1990;18:472-484.

24. Fotherby K. Levonorgestrel: clinical pharmacokinetics. Clin Pharmacokinet 1995;28:203-215.

25. Almeida L, Maia J, Soares-da-Silva P. A double-blind, add-on, placebo-controlled, exploratory trial of eslicarbazepine acetate in patients with partial-onset seizures. Epilepsia 2005;46(Suppl 8): 167-168. 